\title{
H-Ras transfers from B to T cells via tunneling nanotubes
}

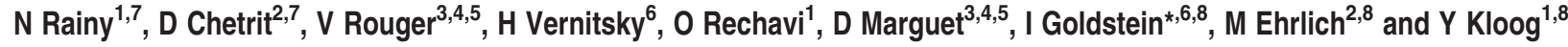

Lymphocytes form cell-cell connections by various mechanisms, including intercellular networks through actin-supported long-range plasma membrane (PM) extensions, termed tunneling nanotubes (TNTs). In this study, we tested in vitro whether TNTs form between human antigen-presenting B cells and T cells following cell contact and whether they enable the transfer of PM-associated proteins, such as green fluorescent protein (GFP)-tagged H-Ras (GFP-H-Ras). To address this question, we employed advanced techniques, including cell trapping by optical tweezers and live-cell imaging by 4D spinning-disk confocal microscopy. First, we showed that TNTs can form after optically trapped conjugated B and T cells are being pulled apart. Next, we determined by measuring fluorescence recovery after photobleaching that GFP-H-Ras diffuses freely in the membrane of TNTs that form spontaneously between B and T cells during coculturing. Importantly, by 4D time-lapse imaging, we showed that GFP-H-Ras-enriched PM patches accumulate at the junction between TNTs and the T-cell body and subsequently transfer to the T-cell surface. Furthermore, the PM patches adopted by T cells were enriched for another B-cell-derived transmembrane receptor, CD86. As predicted, the capacity of GFP-H-Ras to transfer between B and T cells, during coculturing, was dependent on its normal post-transcriptional lipidation and consequent PM anchorage. In summary, our data indicate that TNTs connecting $B$ and T cells provide a hitherto undescribed route for the transfer of PM patches containing, for example, H-Ras from B to T cells. Cell Death and Disease (2013) 4, e726; doi:10.1038/cddis.2013.245; published online 18 July 2013

Subject Category: Immunity

Lymphocytes circulate through the entire body and constantly survey their environment by interacting transiently with many cell types; such dynamic interactions are crucial for the immune response. For example, they form with antigenpresenting cells, including B cells, at a specific cell-cell contact region, the immunological synapse (IS). ${ }^{1,2}$ The IS also induces transient structural continuity between the plasma membrane (PM) of the conjugated cells by membrane fusions and bridges. ${ }^{3}$ For instance, it was reported that membrane nanotubes form de novo between lymphocytes and target cells when the cells move apart after a prolonged tight contact. ${ }^{4,5}$ It can be thus hypothesized that such membrane nanotubes originate from such membrane bridges.

Tunneling nanotubes (TNTs) are transient membrane connections that can facilitate long-range intercellular communication between the linked cells. These structures are dynamic, with lifetimes ranging from minutes up to several hours and a length up to several cell diameters. ${ }^{4,6}$ TNTs were first identified in PC12 cells and subsequently observed in various cell types including immune cells. ${ }^{4,6-9}$ Long-range membrane nanotubes were described to form spontaneously between Jurkat $\mathrm{T}$ cells in vitro, and further analysis revealed that they form de novo among Jurkat cells when a cell conjugate separates and the cells move apart. Interestingly, these typically close-ended TNTs facilitated, for example, the intercellular spread of HIV virions among T cells. ${ }^{5}$ In contrast to trogocytosis (i.e., the snatching of PM fragments at the $1 S^{10}$ ), previous studies of TNTs forming among Jurkat cells did not demonstrate seamless cell-to-cell transfer of PM-associated proteins. ${ }^{5}$

In this regard, in previous studies we have discovered that $\mathrm{H}$-Ras - a small GTPase that undergoes post-translational lipidation and consequently localizes to the inner PM transfers from B721.221 transfectants to $T$ and NK cells. Moreover, the transfer was strictly contact and actin dependent, as this process was inhibited when the donor and acceptor cells were separated by a $0.4-\mu \mathrm{m}$-pore transwell membrane and treated with latrunculin $B$ that inhibits actin polymerization, respectively. ${ }^{11,12}$ Moreover, the rate of transfer correlated with the rate of cell-conjugate formation. ${ }^{12}$

\footnotetext{
'Department of Neurobiology, The George S Wise Faculty of Life Sciences, Tel Aviv University, Tel Aviv 69978, Israel; ${ }^{2}$ Department of Cell Research and Immunology, The George S Wise Faculty of Life Sciences, Tel Aviv University, Tel Aviv 69978, Israel; ${ }^{3}$ Centre d'Immunologie de Marseille-Luminy (CIML), Aix-Marseille University, UM2, Marseille, France; ${ }^{4}$ Institut National de la Santé et de la Recherche Médicale (INSERM), U1104, Marseille, France; ${ }^{5}$ Centre National de la Recherche Scientifique (CNRS), UMR7280, Marseille, France and ${ }^{6}$ Sheba Cancer Research Center, Chaim Sheba Medical Center, Tel Hashomer 52621, Israel; Sackler Faculty of Medicine, Tel Aviv University, Tel Aviv, Israel

${ }^{*}$ Corresponding author: I Goldstein, Sheba Cancer Research Center, Chaim Sheba Medical Center, Tel Hashomer 52621, Israel. Tel: +972 35304901 ; Fax: +972 3 5303506; E-mail: itamar.goldstein@sheba.health.gov.il

${ }^{7}$ These authors contributed equally as first authors.

${ }^{8}$ These authors contributed equally as senior investigators.

Keywords: cellular immunology; cell-cell interactions; intercellular transfer

Abbreviations: APC, allophycocyanin; FACS, fluorescence-activated cell sorting; FRAP, fluorescence recovery after photobleaching; GFP, green fluorescent protein; GPI, glycosylphosphatidylinositol; IS, immunological synapse; PM, plasma membrane; TNTs, tunneling nanotubes

Received 26.4.13; revised 05.6.13; accepted 06.6.13; Edited by G Melino
} 
In a following study, we showed that many other PMassociated proteins transfer among lymphocytes. ${ }^{13}$ More recently, Daubeuf et al. ${ }^{14}$ confirmed our results and showed that Ras family proteins and other inner PM-associated proteins are efficiently transferred from HEK293 transfectants to human T cells.

In this study, our aim was to answer whether the intercellular transfer of membrane-associated proteins, using green fluorescent protein (GFP)-H-Ras as a model for such transfer, can be mediated by TNTs. Here, we report for the first time, by employing advanced technologies (e.g., optical tweezers and 4D spinning-disk confocal microscopy), that TNTs that form between human B721.221 and Jurkat T cells can serve to transfer $\mathrm{H}$-Ras-enriched membrane patches between target cells and $\mathrm{T}$ cells.

\section{Results}

Formation of TNTs upon the separation of cell conjugates. In previous studies, we have discovered that $\mathrm{H}$-Ras, an inner PM-associated GTPase, transfers from 721.221 B cells (B721.221) transfected to stably express GFP-H-RasG12V to $T$ cells, including Jurkat cells. This transfer was strictly contact dependent and was inhibited by compounds that interfere with normal actin polymerization. ${ }^{12}$ In this study, we wanted to determine whether TNTs previously described to form among lymphocytes following tight cell-cell contact can serve to transfer PM patches from B to T cells using GFP-labeled H-RasG12V as a 'model' for membrane-associated proteins.

The proposed model for TNTs' formation between lymphocytes entails prolonged ( $>5 \mathrm{~min}$ ) cell-cell contact that probably induces the initial formation of membrane tunnels between the cells that upon cell-conjugate separation extend to give rise to long-range TNTs. ${ }^{4,5,15}$ Thus, we first asked, using holographic optical tweezers set on an inverted fluorescence microscope-based system, ${ }^{16}$ whether TNTs can be indeed induced to form among Jurkat and B721.221 cells by prolonged cell-cell contact followed by mechanical separation of the cell conjugate (see schematic representation of the experimental design shown in Figure 1A). Thus, we seeded B721.221 cells engineered to stably express GFP-H-RasG12V (B721.221-GFP-H-RasG12V) and Jurkat cells onto Lab-Tek chambered cover glasses. Subsequently, a green B721.221-GFP-H-RasG12V cell and a Jurkat cell were optically trapped and brought together to promote cellcell conjugation (Figure 1Ba and Supplementary Movie 1). After $90 \mathrm{~min}$, the conjugated cells were pulled apart by moving the two cells in individual optical traps that were moved in opposite directions at a mean rate of $\sim 140 \mathrm{~nm} / \mathrm{s}$. In the course of this mechanical cell separation, we observed (in 3 out of 10 experiments) the de novo formation of an intercellular connecting membrane tube of a submicron diameter, which resembled the spontaneous formation of TNTs previously described ${ }^{4,5}$ among lymphocytes following cell-cell contact (Figures $1 \mathrm{Bb}$ and $\mathrm{Bc}$ and Supplementary Movie 2). We also found that such nanotube-like connections induced by mechanically pulling the conjugated cells apart were typically derived from PM extensions of the B721.221 transfectants, as they were labeled throughout with GFP-H-RasG12V (Figure 1C). In a few experiments while optically pulling apart the conjugated cells, we induced the tearing of the B-to-T-cell-connecting nanotube. Under these circumstances, we typically detected GFP-H-RasG12Vlabeled membrane patches of B721.221-cell origin that were retained post-tearing of the nanotube on the red-labeled Jurkat cell (Figure 1D).

These findings are indeed in agreement with the proposed model for TNT formation in lymphocytes, which entails close contact among lymphocytes promoting the formation of PM 'bridges' among the cells that upon cell-conjugate separation develop into long-range TNTs.

Confocal 4D imaging identifies intercellular transfer of GFP-HRasG12V via TNTs. Based on our current findings and previous observations, as described above, we next asked whether TNTs form spontaneously between B721.221 and Jurkat cells and whether they facilitate $\mathrm{H}$-Ras transfer among the two coculture partners. To answer this issue, we turned to 4D spinning-disk confocal microscopy that allows image acquisition at high speed with low light and thus provides ideal imaging conditions for living cells and of relatively weak fluorescent signals. To visualize both cell types by fluorescence microscopy, we stained the Jurkat cells with a lipophilic membrane dye PKH26GL (red emission). Both cell types were plated onto fibronectin-covered Lab-Tek chambered cover glasses and allowed to settle for 90 min prior to visualization by 4D time-lapse imaging.

The unbiased inspection at time 0 (90 min post plating) of multiple fields revealed the acquisition of GFP-H-RasG12Vlabeled membrane patches in a significant percentage of the red-labeled Jurkat cells. Moreover, we identified multiple longrange nanotubes with a length up to several cell diameters, compatible with TNTs, stretching between B721.221 cells and Jurkat cells (Figures 2A-D). We also found that a large percentage of the TNTs that formed between the two cell types were entirely GFP-H-RasG12V-labeled, indicating that they originated from PM extensions of B721.221 cells (Figures $2 \mathrm{~A}$ and $\mathrm{B}$ ). For example, in Figure $2 \mathrm{~A}$, extracted from a time-lapse movie, we show a long-range GFP-H-RasG12V-labeled TNT connecting between a B721.221 and a Jurkat cell (Supplementary Movie 3). Additional example of a similar type of TNT is shown in Figure 3b (a reconstructed 3D image extracted from a 4D time-lapse movie). In Figure $2 \mathrm{C}$, we show an image of different type of TNT that is composed from the PM extensions of both cell types.

In addition, we also detected TNTs that mainly originated from Jurkat cells. For instance, in Figure 2D, we show a PKH26GL (red)-labeled TNT derived from the PM extensions of a red Jurkat cell that networks with two B721.221 cells. In the 4D time-lapse sequences, we captured a GFP-H-RasG12V PM patch of B721.221-cell origin (Figures 2Da-c, see arrows) traveling in the red-labeled TNT toward the Jurkat cell body. Importantly, this membrane patch containing GFP-H-RasG12V molecules was eventually retained on the Jurkat PM even after the tearing of the TNT for an extended time (Figure 2Dd, arrow, and also see Supplementary Movie 4). Reciprocally, we also detected a 
A a
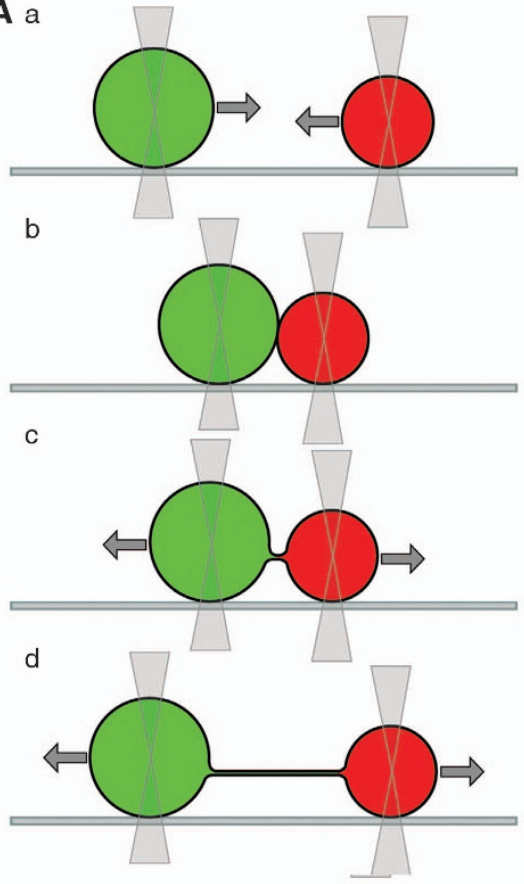

C

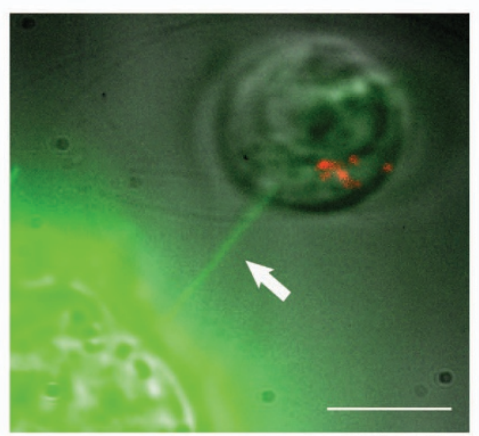

B

a

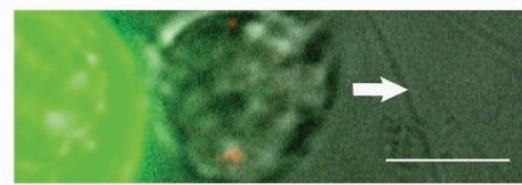

b

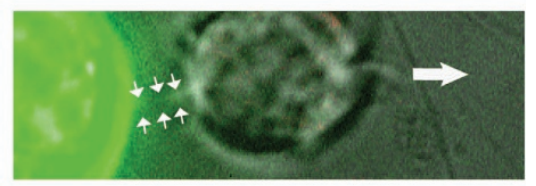

C
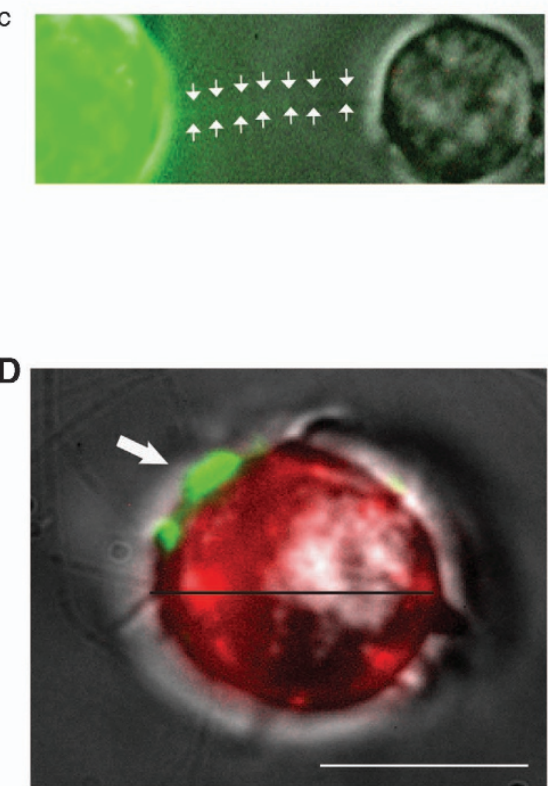

Figure 1 Nanotubes can be induced between B and T cells during the separation of cell conjugates. (A) Schematic representation of the experimental design of the holographic optical tweezers used to optically trap a red-labeled Jurkat cell and GFP-labeled B721.221 cell: (a) to drag them toward each other; (b) to create a cell conjugate; (c) to keep the cell conjugated for $90 \mathrm{~min}$; and (d) then pull them apart in the opposite directions to induce an intercellular connecting TNT. (B) Images were obtained during time-lapse microscopy using a Nikon Intensilight lamp, and the fluorescence emission was captured on an EMCCD camera from Roper Scientific. (a) A cell conjugate created by optically trapping and joining together of a red-labeled Jurkat cell and a B721.221-GFP-H-RasG12V cell (Supplementary Movie 1; the image is from Supplementary Movie 2 before separating the cells). ( $\mathrm{b}$ and $\mathrm{c}$ ) The cells were kept attached for $1.5 \mathrm{~h}$ and then pulled apart to break the cell conjugate. During the separation, an intercellular connecting nanotube was observed to form between the cells (see arrows; the images are from Supplementary Movie 2). (C) An image of a typical nanotube induced, as detailed above, between a B721.221 cell and a Jurkat cell. This nanotube was primarily derived from the PM extension of the B721.221-GFP-H-RasG12V cell. (D) After tearing of the nanotube, we detected GFP-H-RasG12V patches that were transferred to the Jurkat cell. Scale bars represent $5 \mu \mathrm{m}$. The results are representative of a typical experiment out of 10 experiments performed $(n=10)$

PKH26GL-labeled membrane patch of Jurkat T-cell origin that was retained on the formerly connected B721.221-GFP-HRasG12V cells, indicating the existence of a bidirectional transfer (Figure 2D, lower panels).

Next, by continuous 4D time-lapse imaging, we focused on a small area at the junction of a typical B721.221-derived H-RasG12V-labeled TNT and the Jurkat cell body (Figure 3, arrowhead). Zooming in on this junction area, we observed a continuous change in the shape of the initial (see time 0 inset) GFP-H-RasG12V patch that accumulated at this spot. More importantly, we detected small patches (marked in the inset by $\star$ ) that physically separated, in the course of $60 \mathrm{~min}$, from the end of the green-labeled TNT and transferred to the PM of the red Jurkat cell. Taken together, these findings strongly suggest that TNTs represent a hitherto unknown mode for the transfer of membrane patches containing lipidated membrane-anchored $\mathrm{H}$-Ras proteins between lymphocytes.

TNTs facilitate lateral diffusion of H-Ras molecules from B to Jurkat cells. Our previous studies, using fluorescence recovery after photobleaching (FRAP), establish that H-Ras molecules, both wild type and the constitutively active $\mathrm{G} 12 \mathrm{~V}$ mutant, undergo fast lateral diffusion at the PM rather than exchange between membrane and cytoplasmic pools. ${ }^{17}$ Thus, to probe for diffusion of GFP-H-RasG12V in the TNTs and in the membrane patches that typically formed at the junction between the TNT and the Jurkat cell body, we also employed here a FRAP-based approach. In line with our 

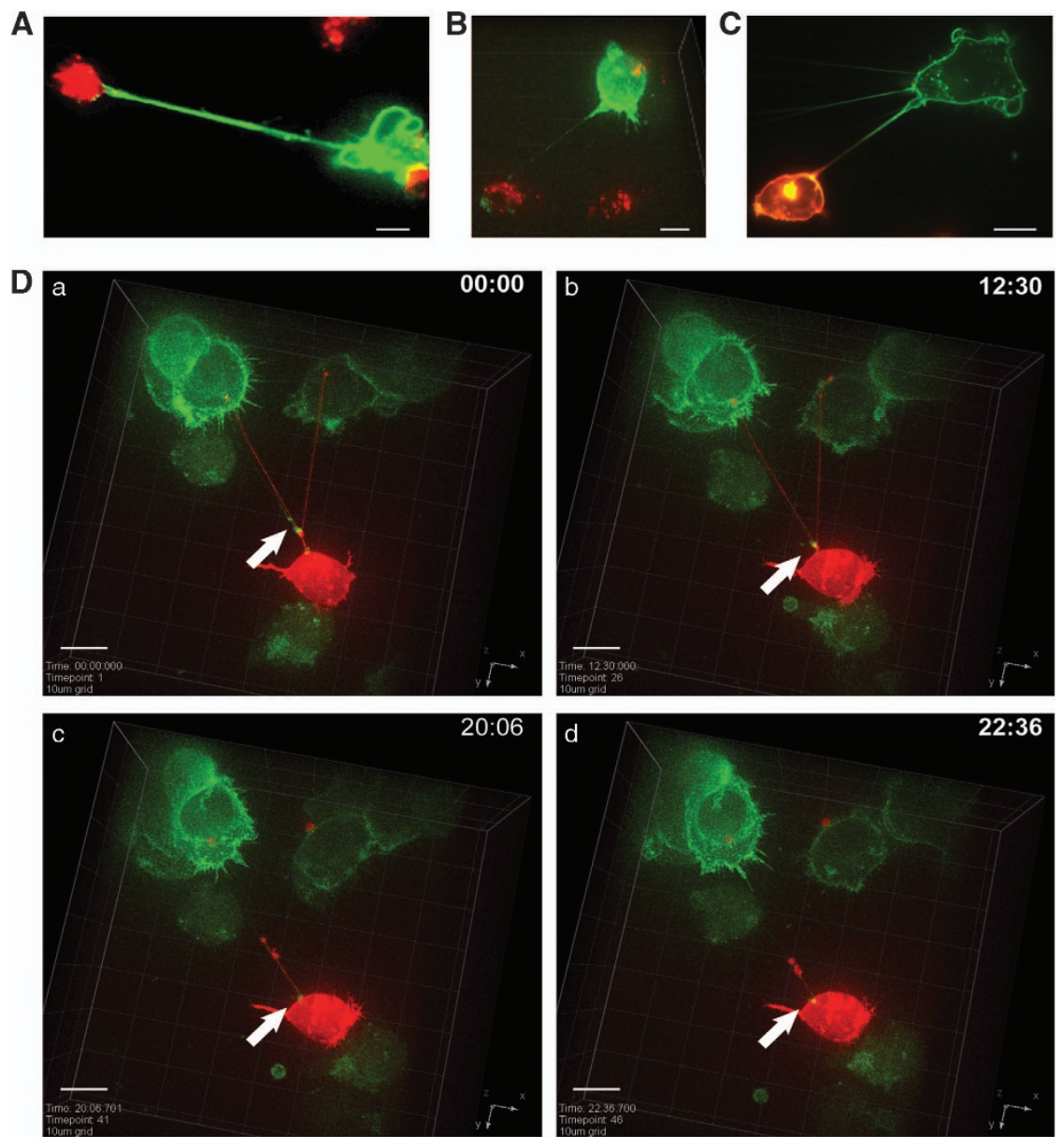

Figure 2 GFP-H-RasG12V transfers from B721.221 to Jurkat cells through TNTs. B721.221 cells expressing GFP-H-RasG12V and Jurkat cells labeled with PKH26GL were seeded onto Lab-Tek chambered cover glasses for $90 \mathrm{~min}$. Subsequently, the cells were imaged by 4D spinning-disk confocal microscopy for an additional $\sim 60$ min. (A) Image from a time-lapse movie (Supplementary Movie 3) showing a long-range GFP-H-RasG12V-labeled TNT connecting between a B721.221 cell and a Jurkat cell. (B) Reconstructed 3D image of another similar TNT extracted from a 4D time-lapse movie. (C) TNT that is composed from the PM extensions of both cell types. (D) The images were extracted from a 4D time-lapse sequence. The sequence depicts the transfer of a GFP-H-RasG12V-enriched (green) membrane patch via the red-labeled TNT from B721.221 cells to a Jurkat cell that is eventually retained on the Jurkat cell's PM even after the tearing of the TNT (see also Supplementary Movie 4). Scale bars represent $5 \mu \mathrm{m}$. The images shown are from representative experiments out of $>10$ experiments performed $(n>10)$
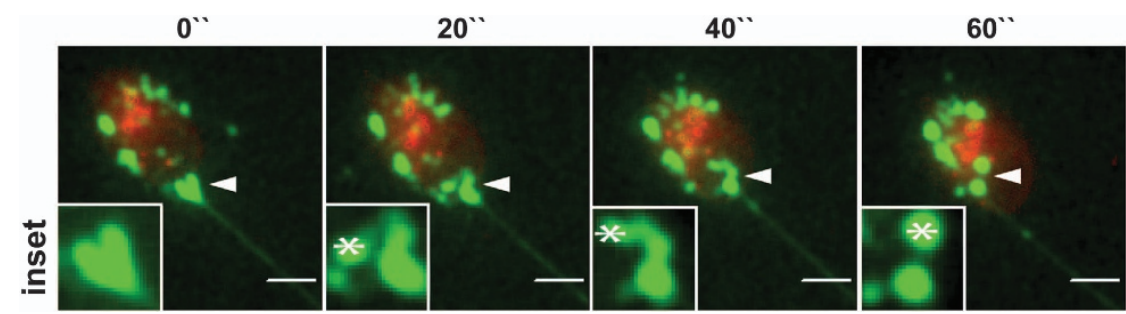

Figure 3 GFP-H-RasG12V accumulates at the junction between TNTs and the Jurkat cell body and subsequently transfers to Jurkat cells. Cells were seeded onto Lab-Tek chambered cover glasses and imaged as detailed above. Images are maximum-intensity projections of confocal z-sections, acquired during continuous (every $80 \mathrm{~s}$ ) 4D spinning-disk confocal microscopy. The images are from a 4D time-lapse sequence depicting the continuous transfer of GFP-H-RasG12V membrane patches at the junction region (indicated by arrowheads) between a TNT derived from the PM extension of a B721.221-GFP-H-RasG12V cell (out of the frame) and the Jurkat cell body. Scale bars represent $3 \mu \mathrm{m}$. Insets show with further detail the dynamic separation and transfer, over time, of discrete GFP-H-RasG12V-enriced membrane patches from the end region of the TNT to the Jurkat cell (marked by *). The results are representative of a typical experiment out of $>3$ experiments performed

previous observations, ${ }^{17}$ the photobleaching of the ventral membrane of the donor B721.221-GFP-H-RasG12V cells resulted in rapid recovery of the GFP signal compatible with lateral membrane diffusion (Figure $4 a$ and curve a in Figure 4b). Photobleaching of a region within GFP-H-RasG12V-labeled TNTs also resulted in good fluorescence recovery, albeit to slightly lower levels and with slower diffusion kinetics (Figure $4 \mathrm{a}$ and curve $\mathrm{b}$ in Figure 4b). In contrast, photobleaching of a typical GFP-H-RasG12V-rich membrane patch at the junction of the TNT and the Jurkat cell body did not result in meaningful fluorescence recovery (Figure $4 a$ and curve $c$ in Figure $4 b$ ). The latter finding suggests the 

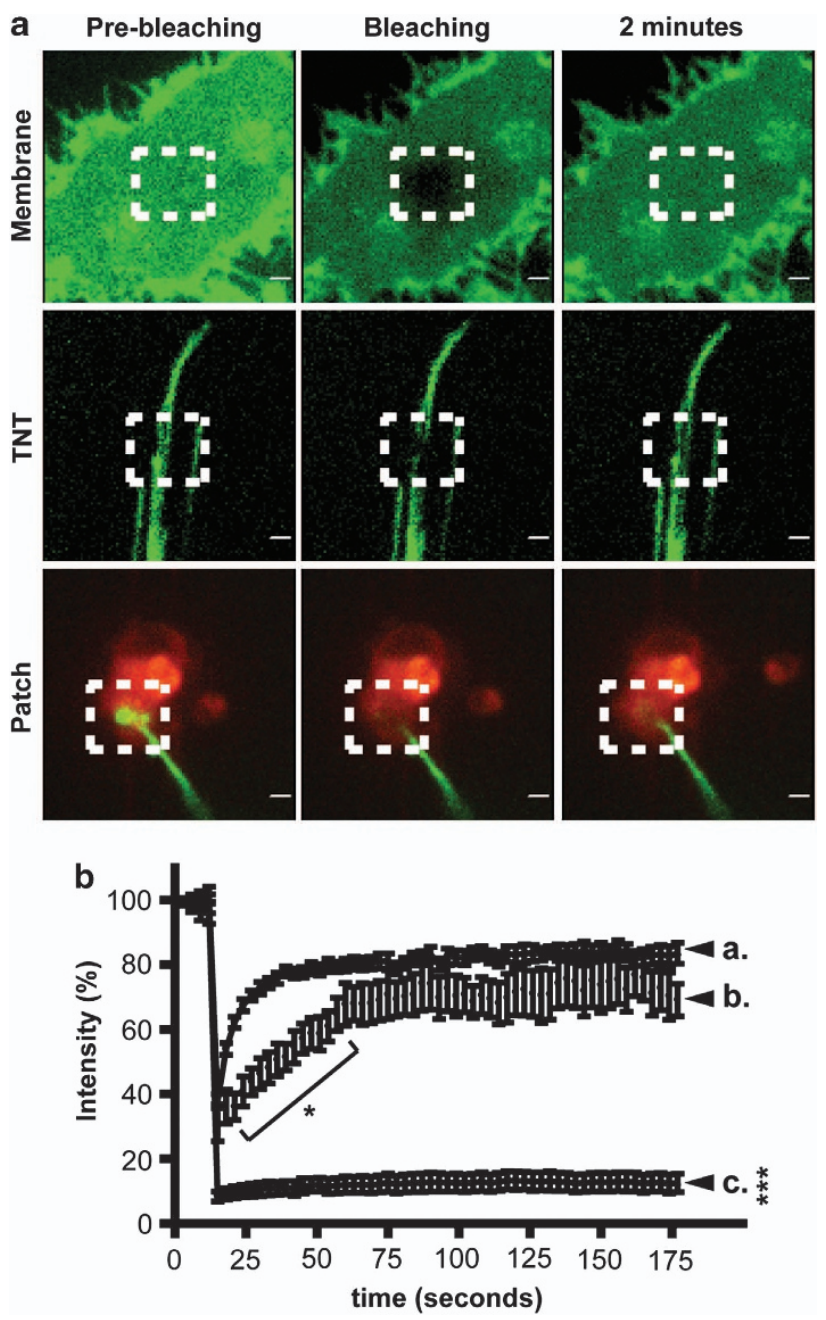

Figure 4 Recovery dynamics of GFP-H-RasG12V differ according to its cellular localization. B721.221 cells expressing GFP-H-RasG12V and PKH26GL red-labeled Jurkat cells were seeded and imaged as detailed in Figure 2. GFP-H-RasG12V was bleached at three different cellular localizations: the ventral membrane of the donor B721.221 cell; the central portion of a B721.221/Jurkat cell-connecting TNT and a GFP-H-Ras-G12V-labeled patch on a Jurkat cell bordering a TNT originating from a B721.221 transfectant. (a) Panels depict typical confocal micrographs prior to bleaching, immediately post-bleaching and after a 2-min period of recovery at each of the cellular localizations. Scale bars represent $1 \mu \mathrm{m}$. (b) Initial fluorescence intensities in FRAP measurements were determined as $100 \%$. Graph depicts the mean \pm S.E.M. of the normalized fluorescence of 10 independent measurements for each of the cellular localizations. At the indicated section of curve $b$ and throughout the entire measurement of curve $c$, the relative fluorescence intensities were significantly different than the analogous sections of curve a, by two-tailed Student's $t$-test $\left({ }^{*} P<0.05,{ }^{* * *} P<0.001\right)$

existence of a barrier that prevents free lateral membrane diffusion between such patches and the TNT, although by 4D spinning-disk confocal microscopy the latter patches appeared to be connected to the TNTs. In some instances, as mentioned before, we observed the tearing of the TNTs in the course of the time-lapse sequence; yet the GFP-H-RasG12V patches were generally retained on the Jurkat cell following this event (Supplementary Movie 4).

Thus, the data presented in this part support the notion that lateral membrane diffusion is a mechanism for $\mathrm{H}$-Ras trafficking within TNTs connecting B721.221 and Jurkat cells. Importantly, the H-Ras-rich membrane patches typically detected at the junction between TNTs and the Jurkat cell body were in fact segregated from the TNTs, and moreover, they remained on the Jurkat cell surface after the tearing of the TNTs.

The transferred PM patches maintain their original in-out orientation. Next, we wanted to determine the topological arrangement of GFP-H-RasG12V within the transferred membrane patches. In this regard, in our previously published studies we observed, by a fluorescenceactivated cell sorting (FACS)-based approach, that GFP-H-RasG12V acquired by $\mathrm{T}$ cells during coculturing was undetectable by immunostaining with anti-Ras and anti-GFP mAbs. However, we found a strong correlation between the level of H-Ras and CD86 (a transmembrane $\mathrm{B}$-cell marker) acquired by $\mathrm{T}$ cells; the latter receptor was easily detectable by staining with specific mAbs. ${ }^{12,13}$ Thus, to further address this latter issue, we immediately immunostained the cells without permeabilization at the end of coculturing using fluorochrome-conjugated mAbs that specifically recognize the extracellular domain of CD86. Our data show that indeed the extracellular domain of this receptor was detectable and colocalized with the B721.221-derived GFP-H-RasG12V-labeled PM patches that were transferred to the Jurkat cells (Figure 5). These latter findings indicate that the transferred PM patches acquired by Jurkat cells retained their correct in-out orientation and localized to the surface of the Jurkat cells.

H-Ras intercellular transfer depends on its farnesylation and palmitoylation. To examine the molecular requirements for the contact-dependent intercellular transfer of $\mathrm{H}$-Ras from $\mathrm{B}$ to $\mathrm{T}$ cells, regardless of its mechanism, we made use of $\mathrm{H}$-RasG12V mutants, differing in their ability to undergo post-translational lipid modifications. H-Ras processing involves the addition of one farnesyl and two palmitoyl molecules into its C-terminal tail. ${ }^{18,19}$ Farnesylation that occurs on $\mathrm{C} 186$ is absolutely required for further $\mathrm{H}$-Ras processing by palmitoylation on $\mathrm{C} 181$ and $\mathrm{C} 184 .^{20}$ To probe for the roles of these post-translational modifications in the recruitment of GFP-H-RasG12V to the PM of B721.221 cells, we generated cell lines stably expressing GFP-H-RasG12V with different $\mathrm{C}$-terminal mutations. The cells were then fixed, permeabilized and immunostained with anti-GM130 Abs (Golgi complex, red channel), and the nuclei were labeled with DAPI. Analysis by confocal microscopy confirmed previous observations ${ }^{21,22}$ and showed that all lipid modifications are necessary for optimal recruitment of GFP-H-RasG12V to the PM (Figure 6a). We found that the C184S mutant localized both to the Golgi and the PM and the C181S showed aberrant localization to the cytosol, the Golgi complex and the PM. The mutated C186S (no lipidation) showed a predominant cytosolic localization, and C181S/ C184S (no palmitoylation) was localized to the cytosol and the Golgi complex. In contrast, the unmodified H-RasG12V was almost exclusively localized to the PM (Figure 6a). These results are in agreement with previous studies. ${ }^{20-22}$ Next, we tested whether these mutations that regulate PM 


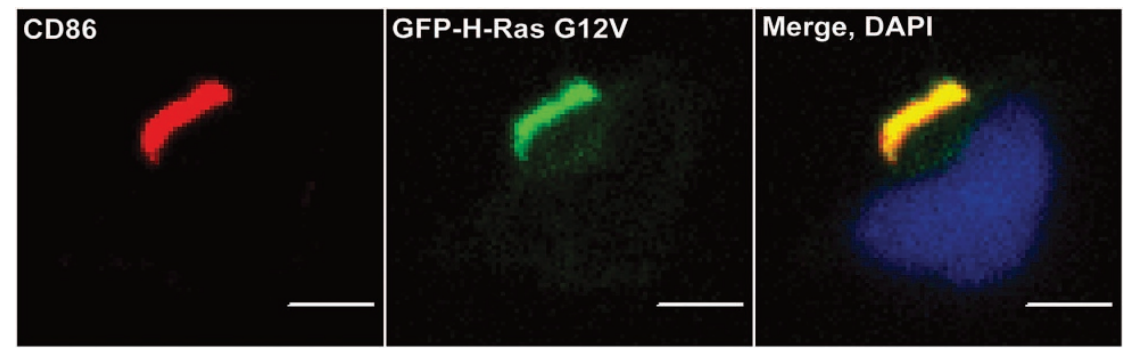

Figure 5 Transferred GFP-H-RasG12V-enriched PM patches maintain proper in-out orientation. B721.221 cells expressing GFP-H-RasG12V and Jurkat cells labeled with CellVue Claret Far Red Fluorescent dye were seeded as detailed above. Two hours post seeding, cells were fixed and stained with mAbs that specifically recognize the extracellular domain of CD86, followed by staining with Alexa Fluor 555-conjugated secondary antibodies. Panels (single colored and merged) depict a single confocal section of a typical Jurkat cell decorated with a GFP-H-RasG12V patch (scale bars represent $5 \mu \mathrm{m}$ )

anchorage modulate the intercellular transfer of GFP$\mathrm{H}$-RasG12V to $\mathrm{T}$ cells. To address this question in a quantitative approach, we cocultured the different B721.221 transfectants with activated peripheral blood-derived T cells. By multi-parametric digital flow cytometry analysis, combined with a stringent gating algorithm (see the Materials and methods section for further details), we distinguished the viable singlet $T$ cells from $B / T$-cell conjugates and determined their GFP content. We found (Figures $6 b$ and $c$ ) that the intercellular transfer of the GFP-H-RasG12V/C186S and GFP-H-RasG12V/C181S/C184S mutants, which lack palmitoylation and displayed a mainly cytosolic pattern of distribution, was most significantly impaired relative to GFP-H-RasG12V $(87 \pm 0.94 \%$ and $85 \pm 3.3 \%$ reduction in transfer, respectively, $P<0.01$, by the non-parametric Mann-Whitney $U$ test). In contrast, the single mutants GFP-H-RasG12V/C181S and GFP-H-RasG12V/C184S that undergo mono-palmitoylation showed a less pronounced reduction in intercellular transfer compared with GFP-H-RasG12V (50 $\pm 1.3 \%$ and $25 \pm 4.5 \%$, respectively, $P<0.01)$. These results, together with the confocal microscopy analysis of the intracellular distribution of the different GFP-H-RasG12V constructs, show that an increase in the cytosolic localization of GFP-H-RasG12V, stemming from reduced post-translational palmitoylation, impairs its intercellular transfer potential.

\section{Discussion}

The main novel findings of this study are that TNTs are a mechanism of intercellular transfer of $\mathrm{H}$-Ras-containing membrane patches from antigen-presenting $B$ cells to $T$ cells and that proper $\mathrm{H}$-Ras lipidation and $\mathrm{PM}$ anchorage are necessary for optimal intercellular transfer.

The results of our FRAP experiments show that $\mathrm{H}$-RasG12V enters and traffics in TNTs by lateral membrane diffusion. Our data suggest a significantly slower rate of recovery ( $\sim 4-5$-fold slower) of the GFP-H-RasG12V signal in the TNTs compared with its recovery in the ventral PM of the donor B721.221 cells after photobleaching. Interestingly, Sowinski et al. ${ }^{5}$ detected similar rates of FRAP for a glycosylphosphatidylinositol (GPI)-anchored GFP both in the TNTs connecting Jurkat cells and on the Jurkat cell surface. These findings raise the possibility that interactions of $\mathrm{H}$-Ras with domains found in the inner leaflet of the TNTs' membranes restrict its lateral diffusion, whereas such constrains are less evident for GPI-anchored proteins attached to the external leaflet of the PM.

Importantly, within GFP-H-RasG12V-enriched membrane patches at the junction between the TNT and the Jurkat cell body, FRAP was undetectable. The lack of recovery of the GFP-H-RasG12V signal in these B721.221-derived PM patches proves that they are in fact segregated from the rest of the TNT. Moreover, we found by immunostaining of unpermeabilized cells that post coculturing GFP-H-RasG12V colocalizes with the B-cell surface-specific receptor CD86 in such distinct PM patches that have been retained on the Jurkat cell surface. Our results also show that such TNT-derived membrane patches on Jurkat cells do not undergo immediate endocytosis or other form of internalization after the tearing of the TNTs.

Based on the experiments using holographic optical tweezers to trap and move live cells and our coculture system together with previous data summarized in comprehensive reviews, ${ }^{4,11,23}$ we suggest the following integrative model for $\mathrm{H}$-Ras transfer. Initially, during the formation of an intimate close contact between B and T cells, H-Ras is directly transferred to the acceptor $T$ cell in a process that resembles trogocytosis, that is, the snatching of PM patches by lymphocytes during the formation of an IS. Upon separation of the $B / T$-cell conjugate, the cells maintain long-range contacts through fine PM-derived extensions, termed nanotubes, which elongate as the intercellular distance grows. In parallel, these TNTs facilitate the lateral diffusion of $\mathrm{H}$-Ras and other PM-anchored proteins, such as CD86. This results in the accumulation of distinct membrane patches at the junction of the TNTs and the Jurkat cell body, which eventually disconnect and localize to the surface of the $\mathrm{T}$ cell. This observed sequence of events is consistent with previous observations reported by the Davis $\mathrm{Lab}^{4,5}$ that imply that TNTs forming among Jurkat cells generally have a closeended dynamic junction that could be viewed as a 'nano-scale' IS. Although in this latter study the authors did not observe seamless transfer of transmembrane proteins between connected Jurkat cells, it can be envisioned that when TNTs form between antigen-presenting cells and $T$ cells, this dynamic 'nano-scale' IS that forms at the junction region can facilitate the snatching of membrane patches by $T$ cells - in a yet undefined cellular process. In agreement with our observations, Arkwright et al. have previously shown that 


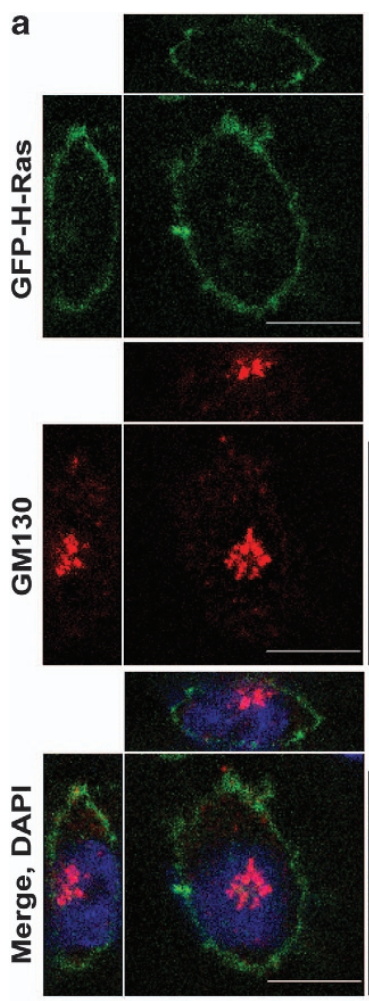

H-RasG12V
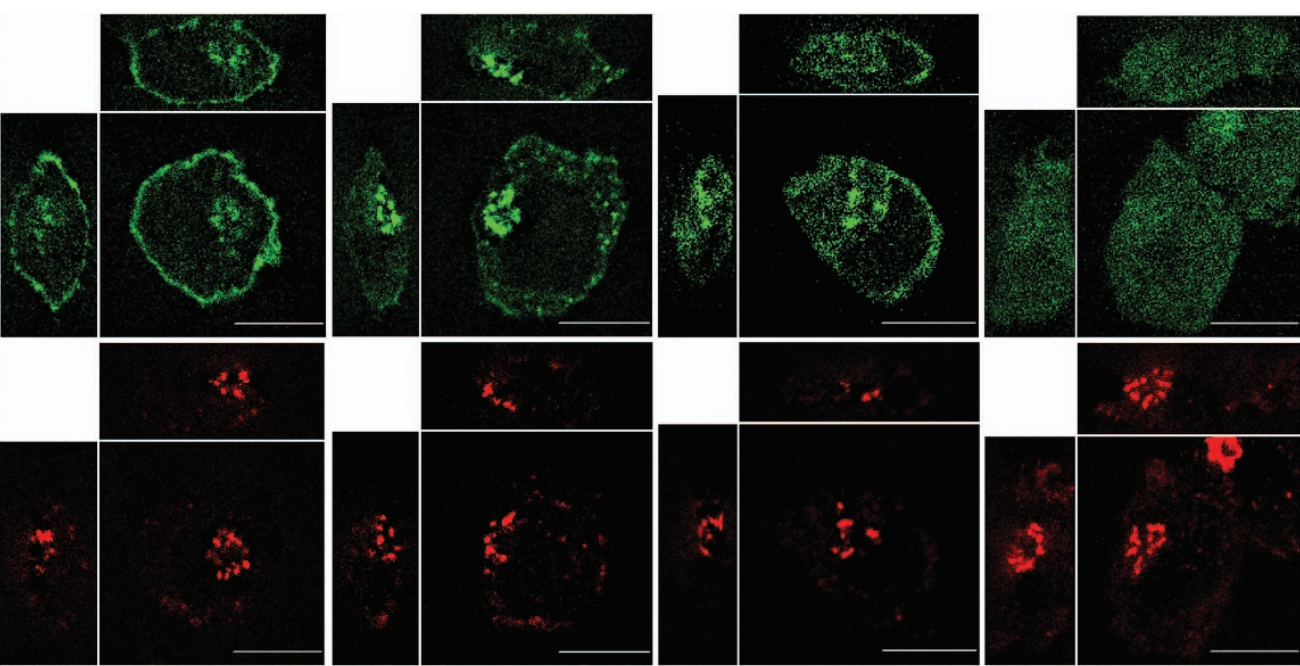

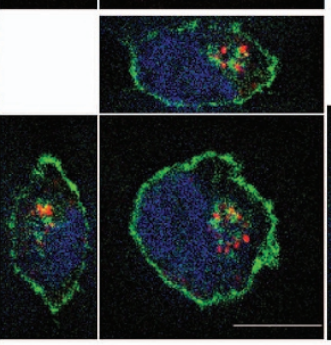

C181S

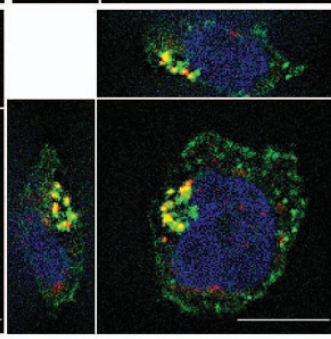

C184S

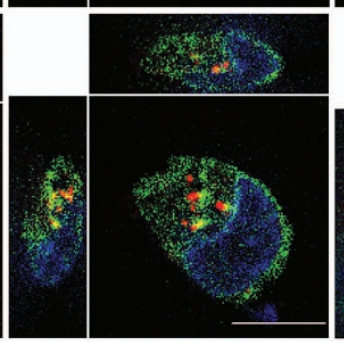

C181S/C184S

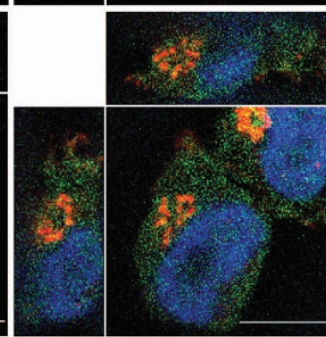

C186S b
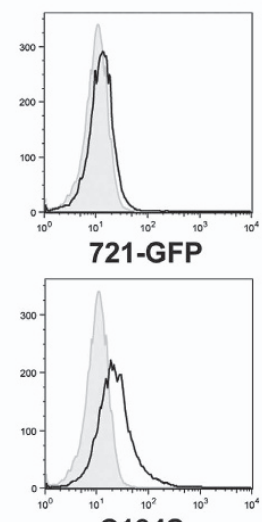

C184S

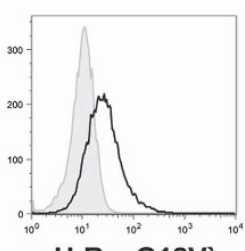

H-RasG12V

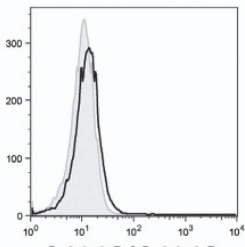

C181S/C184S

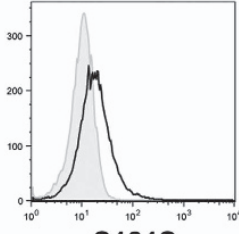

C181S

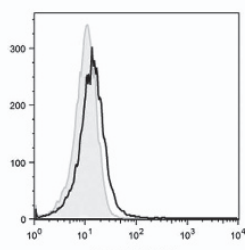

C186S

C

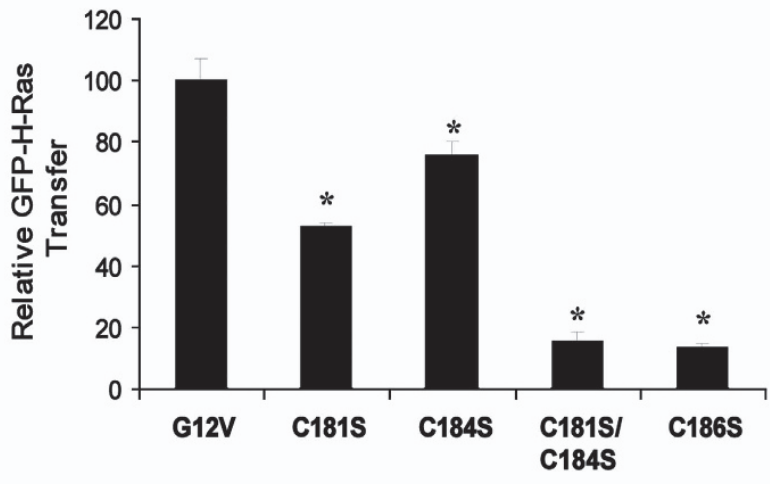

Figure 6 H-Ras intercellular transfer depends on its farnesylation and palmitoylation. (a) Cells were plated on a Lab-Tek chambered cover glass, and the entire cell volume was captured through successive $z$-sections using spinning-disk confocal microscopy. Panels depict a confocal mid-section ( $x, y$ dimensions, large central square) as well as $x$, $z$ and $y, z$ cross-sections (smaller lateral rectangles) of the different GFP-H-RasG12V mutants in typical B721.221 stable transfectants. For GFP-H-RasG12V and GM130 colocalization, cells were fixed and permeabilized and labeled with rabbit anti-GM130 Abs as detailed in the Materials and methods section. Images were deconvoluted with NearestNeighbors algorithm (Slidebook) for greater clarity. Scale bars represent $10 \mu \mathrm{m}$. (b) $\mathrm{CD}^{+} \mathrm{T}$ cells were cocultured with the indicated B721.221 transfectants, expressing GFP-H-RasG12V with or without mutations in its C-terminal region, as detailed in the Materials and methods section. The various histogram overlays depict GFP signal detected in T cells (CD3 ${ }^{+}$singlet-cell gate) cocultured with the indicated B721.221 transfectants (black line) versus T cells cultured alone (gray-shaded histogram). (c) Bar chart represents the relative percentage of transfer: that is, relative mean fluorescence intensity of GFP signal detected in T cells cocultured with the various H-RasG12V mutants compared with that in T cells cocultured with the positive control B721.221-GFP-H-RasG12V cells (the latter set as $100 \%$ transfer). Bars represent mean \pm S.E.M., and ${ }^{*} P<0.01$ for all mutants compared with the unmodified GFP-H-RasG12V (comparing the relative GFP-H-Ras transfer values by the non-parametric Mann-Whitney $U$ test). Data are from three independent experiments performed in duplicates or triplicates

TNTs can facilitate seamless transfer of membrane elements among Jurkat $T$ cells in a process that was dependent on Fas signaling. Thus, TNTs were primarily involved in rapidly transmitting Fas-dependent apoptotic signals to adjacent cells, including active caspase- 3 and membrane-anchored Fas ligand. The authors furthermore suggest that this
TNT-mediated transfer of apoptotic signals can promote the spread of 'fratricidal' death. ${ }^{24}$

Our data also show that PM localization is essential for optimal Ras intercellular transfer during coculturing. We found an inverse correlation between aberrant localization to the cytosol of the various H-RasG12V mutants, defective in 
post-translational lipidation and their capacity to transfer. For example, the C186S (no lipidation) and the C181S/C184S (no palmitoylation) $\mathrm{H}-$ RasG12V mutants displayed prominent cytosolic localization, and consequently their capacity to transfer among B721.221 and $\mathrm{T}$ cells was significantly abolished. In contrast, the C184S mutant that is both farnesylated and mono-palmitoylated at C181, and subsequently does not show prominent cytosolic localization, displayed a much smaller reduction in its capacity for intercellular transfer. As we could not detect any meaningful transfer of the C186S and the C181S/C184S mutants, it can be postulated that B-to-T-cell transfer of Ras both at the IS (trogocytosis like) and via TNTs is linked to adequate membrane anchorage. In this regard, Rustom et al. ${ }^{6}$ in their seminal description of TNTs have shown that neither cytoplasmic GFP nor the small dye calcein transfers among PC12 cells via tubular TNTs, although attaching GFP to the $\mathrm{C}$-terminal tail of $\mathrm{H}$-Ras to promote its localization to the PM was strongly linked with cell-to-cell transfer of the lipidated GFP via TNTs. Moreover, it is well known that the transfer of proteins via trogocytosis (i.e., membrane snatching by lymphocytes) is absolutely dependent on their PM association. Thus, the relevant literature and our present data support the conclusion that PM localization is indeed vital for $\mathrm{H}$-Ras transfer among lymphocytes.

Many studies have already established that the intercellular transfer of transmembrane proteins from target cells to lymphocytes and other immune cells ${ }^{23,25,26}$ has important functional consequences. In this regard, in previous studies, we have demonstrated that the transfer of the oncogenic GTP-bound constitutively active H-RasG12V is associated with increased $p$-ERK1/2 levels in the acquiring lymphocytes. In addition, it has been shown that cell-surface receptors acquired by lymphocytes from target cells retain their normal in-out transmembrane orientation in the adopting cell. ${ }^{12,23,25}$ We have previously validated this observation regarding the transfer of GFP-H-Ras from B721.221 cells to NK cells ${ }^{12}$ as well as from melanoma transfectants to T cells. ${ }^{27}$ In these studies, by immunofluorescence staining with anti-Ras mAbs, we found a positive correlation between the immunostaining and the amount of GFP-H-RasG12V acquired by the T cells only in permeabilized cells - but not in intact cells. Moreover, our previous ${ }^{12,27}$ and present findings show that GFP-H-RasG12V is primarily distributed in the PM of the adopting $T$ cells. Taken together, all these multimodality data suggest that the transferred GFP-H-RasG12V is in fact incorporated into the inner aspect of the PM of the adopting $T$ cells and signals to induce the phosphorylation of its downstream effector ERK1/2. In a very recent study, we have furthermore demonstrated that the transfer of H-RasG12V from melanoma cells to melanoma-specific cytotoxic $T$ cells augments their effector functions, including cytokine secretion and cytotoxic degranulation. ${ }^{27}$ However, with current technologies it is difficult to determine the relative functional contribution of membrane-associated H-RASG12V proteins transferring either via TNTs or via trogocytosis.

In conclusion, our present results show that long-range connections via TNTs between $B$ and T cells can facilitate the transfer of $\mathrm{H}$-Ras - a small GTPase anchored to the inner leaflet of the PM-employed in this study as a proof of concept. These novel findings identify TNTs as a route for the intercellular transfer of PM-anchored $\mathrm{H}$-Ras proteins from $\mathrm{B}$ to $\mathrm{T}$ cells.

\section{Materials and Methods}

Human subjects. This study was approved by the Institutional Ethics Committee at the Chaim Sheba Medical Center. Peripheral blood samples were obtained from healthy blood donors who signed an informed consent.

Cell lines. The human Jurkat cell line and the human B lymphoblastoid cell line 721.221 were obtained from American Type Culture Collection (ATCC, Manassas, VA, USA). The genetically engineered stable transfectants B271.221-GFP-HRasG12V and B271.221-GFP were generated as previously described. ${ }^{12}$ All cell lines were cultured in complete RPMI-1640 medium supplemented with $10 \%$ fetal bovine serum, $2 \mathrm{mM} \mathrm{L-glutamine,} 100 \mathrm{U} / \mathrm{ml}$ penicillin and $100 \mu \mathrm{g} / \mathrm{ml}$ streptomycin (all from Gibco, Carlsbad, CA, USA) and were maintained at $37^{\circ} \mathrm{C}$ in a humidified $5 \% \mathrm{CO}_{2}$ incubator. For the optical tweezers experiments and the $4 \mathrm{D}$ imaging experiments, the cells were grown in RPMI-1640 medium, as described above, but without phenol red to reduce its effect on fluorescence.

Antibodies and reagents. Allophycocyanin (APC)-conjugated mouse mAbs directed against human CD3 were obtained from eBioscience (San Diego, CA, USA). The following reagents were purchased from Sigma (St. Louis, MO, USA) PKH26GL red membrane dye and the CellVue Claret Far Red Fluorescent dye, DAPI and anti-GM130 Abs to the Golgi complex. The secondary antibody Alexa Fluor 555-conjugated goat anti-rabbit IgG was from Life Technologies (Grand Island, NY, USA).

Plasmids and stable transfection. The expression vectors containing GFP-H-RasG12V, GFP-H-RasG12V/C181S, GFP-H-RasG12V/C184S and GFP$\mathrm{H}$-RasG12V/C181S/C184S were previously described (Roy et al. ${ }^{22}$ ). Cysteine 186 on GFP-H-RasG12V was changed to serine to create GFP-H-RasG12V/C186S using a specific set of primers (5'-GCATGAGCTGCAAGTCTGTGCTCTCCTG AGG and 5'-CCTCAGGAGAGCACAGACTTGCAGCTCATGC) and Quickchange site-directed mutagenesis kit (Stratagene, La Jolla, CA, USA) according to the manufacturer's protocol, and all vectors were verified by sequencing. B721.221 cells were transfected with the relevant plasmid using Amaxa electroporation system and selected to stably express the indicated vector as previously described. $^{28}$

Optical tweezers experiments. The holographic optical tweezers were generated by an IF laser beam $(1053 \mathrm{~nm})$ focused through a $\times 1001.3 \mathrm{NA}$ oil-immersion objective of an inverted microscope (from Nikon, Tokyo, Japan). The reflection of the laser on the spatial light modulator generates multiple diffracted traps that can be moved in three dimensions and real time by refreshing the hologram at a frequency of $30 \mathrm{~Hz}$, as previously described. ${ }^{16}$ A Nikon Intensilight lamp was filtered to excite the sample at appropriate wavelengths. The fluorescence emission was captured through a $\times 1.5$ tube lens on an EMCCD camera (from Roper Scientific, Martinsried, Germany).

In each experiment, a B721.221 transfectant and a Jurkat cell were optically trapped and dragged using the optical tweezers to create a two-cell conjugate. The cell conjugate was kept in the trap by applying low optical tweezers force. After $90 \mathrm{~min}$, the tweezers force was increased to maximum, and the cells were pulled in opposite directions to break the cell conjugate.

Cell imaging and data acquisition. B721.221 cells stably expressing GFP-H-RasG12V and Jurkat cells prelabeled with the far red fluorescent membrane linker PKH26 were seeded onto a Lab-Tek chambered cover glass (Nunc, Thermo Fisher Scientific, Pittsburgh, PA, USA), precoated with fibronectin (Sigma). Images were acquired using a motorized spinning-disk confocal microscope (Yokogawa CSU-22, Zeiss Axiovert 200M, Jena, Germany). The confocal illumination was with $40 \mathrm{~mW} 473 \mathrm{~nm}$ and $10 \mathrm{~mW} 561 \mathrm{~nm}$ solid-state lasers. Images were acquired with a $\times 100$ oil-immersion objective and Evolve EMCCD camera (Photometrics, Tucson, AZ, USA; $1 \times 1$ binning, yielding a pixel size of $0.16 \mu \mathrm{m}$ ) with typical exposures of $100-500 \mathrm{~ms}$. Three-dimensional image stacks were acquired by sequential acquisition of views recorded along the $z$ axis by varying the position of a piezo electrically controlled stage (typical step size of $0.8 \mu \mathrm{m}$ ). All images were analyzed with SlideBook software (version 5.0; Intelligent Imaging Innovations, Denver, CO, USA). 
For GFP-H-RasG12V and GM130 colocalization, cells were immunostained as previously described. ${ }^{29}$ Briefly, after fixation ( $2 \%$ paraformaldehyde), cells were permeabilized (0.2\% Triton X-100), labeled with rabbit anti-GM130 $(0.2 \mu \mathrm{g} / \mathrm{ml})$ and Alexa Fluor 555-conjugated goat anti-rabbit lgG and imaged at a plane with distinct GM130 Golgi complex labeling.

Photobleaching assay. FRAP was performed with a near-diffraction-limited collimated laser beam (Micropoint, Photonics Instruments, Saint Charles, IL, USA), wavelength tuned with a coumarin-based dye to specifically photobleach GFP. The photobleached area was determined on the computer screen with SlideBook software, and bleaching was achieved with the minimal intensity to avoid possible phototoxicity.

Cocultures and analysis of GFP-H-RasG12V intercellular transfer by FACS. The experiments were performed as previously described. ${ }^{12}$ Briefly, $5 \times 10^{5}$ B721.211 transfectants and $1 \times 10^{6}$ peripheral blood-derived T cells, from healthy blood donors, were incubated in $500 \mu \mathrm{l}$ of complete culture medium in a U-shaped plastic FACS tube (BD Falcon, Tewksbury, MA, USA) to obtain an effector-to-target ratio of $2: 1$. To promote cell-conjugate formation, the cells were centrifuged at $200 \times g$ for $2 \mathrm{~min}$ and then cocultured for $3 \mathrm{~h}$ at $37^{\circ} \mathrm{C}$. For flow cytometry analysis of GFP-H-RasG12V transfer, the cells were stained with antiCD3-APC mAbs for $15 \mathrm{~min}$ at room temperature. Subsequently, the cells were washed, resuspended in $5 \mathrm{mM}$ PBS/EDTA, vortexed and kept on ice to disrupt cell conjugates and produce a single-cell suspension.

FACS analysis. For multi-parametric FACS analysis, cell samples were acquired/analyzed on a FACSCalibur using Cellquest software or on a FACSAria instrument using FACSDiva software (all from BD Biosciences, San Jose, CA, USA). Data were collected from $\sim 10000$ single-cell events. T-cell single-cell events were distinguished from target cells by their by CD3 staining and their FSC/SSC characteristics, as previously published. ${ }^{12,13}$ Final data analysis was performed using the Flow-Jo 7.2.1 software (Tree Star, Ashland, OR, USA).

\section{Conflict of Interest}

The authors declare no conflict of interest.

Acknowledgements. This study was supported in part by grants from the Israel Cancer Association (to IG and YK) and the Israel Science Foundation project $662 / 10$ (to IG and YK). YK is the incumbent of The Jack H Skirball Chair for Applied Neurobiology. This study was performed in partial fulfillment of the requirements for a Ph.D. degree of Nir Rainy (The George S Wise Faculty of Life Sciences, Tel Aviv University, Tel Aviv, Israel). We thank Dr. Yaniv Lerenthal for assistance with imaging data analysis and helpful discussions.

\section{Author contributions}

NR conceived, designed and performed research, collected and analyzed data and wrote the paper. DC designed and performed research and collected and analyzed data. VR performed experiments and collected and analyzed data. HV performed experiments and collected data. OR conceived and designed research and performed experiments. DM designed research and analyzed data. IG conceived and designed research, analyzed data and wrote the paper. ME designed research, analyzed data and wrote the paper. YK conceived and designed research and wrote the paper.

1. Grakoui A, Bromley SK, Sumen C, Davis MM, Shaw AS, Allen PM et al. The immunological synapse: a molecular machine controlling T cell activation. Science 1999; 285: 221-227.

2. Carlin LM, Eleme K, McCann FE, Davis DM. Intercellular transfer and supramolecular organization of human leukocyte antigen $\mathrm{C}$ at inhibitory natural killer cell immune synapses. J Exp Med 2001; 194: 1507-1517.

3. Stinchcombe JC, Bossi G, Booth S, Griffiths GM. The immunological synapse of CTL contains a secretory domain and membrane bridges. Immunity 2001; 15: 751-761.

4. Davis DM, Sowinski S. Membrane nanotubes: dynamic long-distance connections between animal cells. Nat Rev Mol Cell Biol 2008; 9: 431-436.
5. Sowinski S, Jolly C, Berninghausen O, Purbhoo MA, Chauveau A, Kohler K et al. Membrane nanotubes physically connect $T$ cells over long distances presenting a novel route for HIV-1 transmission. Nat Cell Biol 2008; 10: 211-219.

6. Rustom A, Saffrich R, Markovic I, Walther P, Gerdes HH. Nanotubular highways for intercellular organelle transport. Science 2004; 303: 1007-1010.

7. Gousset K, Schiff E, Langevin C, Marijanovic Z, Caputo A, Browman DT et al. Prions hijack tunnelling nanotubes for intercellular spread. Nat Cell Biol 2009; 11: 328-336.

8. Onfelt B, Nedvetzki S, Benninger RK, Purbhoo MA, Sowinski S, Hume AN et al. Structurally distinct membrane nanotubes between human macrophages support long-distance vesicular traffic or surfing of bacteria. J Immunol 2006; 177: 8476-8483.

9. Watkins SC, Salter RD. Functional connectivity between immune cells mediated by tunneling nanotubules. Immunity 2005; 23: 309-318.

10. Joly E, Hudrisier D. What is trogocytosis and what is its purpose? Nat Immunol 2003; 4: 815.

11. Rechavi $\mathrm{O}$, Goldstein I, Kloog Y. Intercellular exchange of proteins: the immune cell habit of sharing. FEBS Lett 2009; 583: 1792-1799.

12. Rechavi $\mathrm{O}$, Goldstein I, Vernitsky H, Rotblat B, Kloog Y. Intercellular transfer of oncogenic H-Ras at the immunological synapse. PLoS One 2007; 2: e1204.

13. Rechavi O, Kalman M, Fang Y, Vernitsky H, Jacob-Hirsch J, Foster LJ et al. Trans-SILAC: sorting out the non-cell-autonomous proteome. Nat Methods 2010; 7: 923-927.

14. Daubeuf S, Aucher A, Bordier C, Salles A, Serre L, Gaibelet G et al. Preferential transfer of certain plasma membrane proteins onto $T$ and $B$ cells by trogocytosis. PLoS One 2010; 5: e8716

15. Chauveau A, Aucher A, Eissmann P, Vivier E, Davis DM. Membrane nanotubes facilitate long-distance interactions between natural killer cells and target cells. Proc Natl Acad Sci USA 2010; 107: 5545-5550.

16. Monneret S, Belloni F, Marguet D. Practical lab tool for living cells based on microstereolithography and multiple dynamic holographic optical tweezers. Proceedings of SPIE 6088, Imaging, Manipulation, and Analysis of Biomolecules, Cells, and Tissues IV 2006; 60880Z.

17. Niv H, Gutman O, Kloog Y, Henis YI. Activated K-Ras and H-Ras display different interactions with saturable nonraft sites at the surface of live cells. J Cell Biol 2002; 157: 865-872.

18. Shields JM, Pruitt K, McFall A, Shaub A, Der CJ. Understanding Ras: 'it ain't over 'til it's over'. Trends Cell Biol 2000; 10: 147-154.

19. Cox AD, Der CJ. Ras history: the saga continues. Small GTPases 2010; 1: 2-27.

20. Choy E, Chiu VK, Silletti J, Feoktistov M, Morimoto T, Michaelson D et al. Endomembrane trafficking of ras: the CAAX motif targets proteins to the ER and Golgi. Cell 1999; 98: 69-80.

21. Hancock JF. Ras proteins: different signals from different locations. Nat Rev Mol Cell Biol 2003; 4: 373-384.

22. Roy S, Plowman S, Rotblat B, Prior IA, Muncke C, Grainger S et al. Individual palmitoyl residues serve distinct roles in $\mathrm{h}$-ras trafficking, microlocalization, and signaling. Mol Cell Biol 2005; 25: 6722-6733.

23. Davis DM. Intercellular transfer of cell-surface proteins is common and can affect many stages of an immune response. Nat Rev Immunol 2007; 7: 238-243.

24. Arkwright PD, Luchetti F, Tour J, Roberts C, Ayub R, Morales AP et al. Fas stimulation of T lymphocytes promotes rapid intercellular exchange of death signals via membrane nanotubes. Cell Res 2010; 20: 72-88.

25. McCann FE, Eissmann $P$, Onfelt $B$, Leung $R$, Davis DM. The activating NKG2D ligand MHC class I-related chain A transfers from target cells to NK cells in a manner that allows functional consequences. J Immunol 2007; 178: 3418-3426.

26. Qureshi OS, Zheng Y, Nakamura K, Attridge K, Manzotti C, Schmidt EM et al, Trans-endocytosis of CD80 and CD86: a molecular basis for the cell-extrinsic function of CTLA-4. Science 2010; 332: 600-603.

27. Vernitsky H, Rechavi O, Rainy N, Besser MJ, Nagar M, Schachter J et al. Ras oncoproteins transfer from melanoma cells to $\mathrm{T}$ cells and modulate their effector functions. J Immunol 2012; 189: 4361-4370.

28. Mandelboim O, Reyburn HT, Vales-Gomez M, Pazmany L, Colonna M, Borsellino G et al. Protection from lysis by natural killer cells of group 1 and 2 specificity is mediated by residue 80 in human histocompatibility leukocyte antigen $C$ alleles and also occurs with empty major histocompatibility complex molecules. J Exp Med 1996; 184: 913-922.

29. Eisenberg S, Beckett AJ, Prior IA, Dekker FJ, Hedberg C, Waldmann $\mathrm{H}$ et al. Raft protein clustering alters N-Ras membrane interactions and activation pattern. Mol Cell Biol 2011; 31: $3938-3952$.

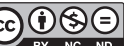

Cell Death and Disease is an open-access journal published by Nature Publishing Group. This work is licensed under a Creative Commons Attribution-NonCommercialNoDerivs 3.0 Unported License. To view a copy of this license, visit http://creativecommons.org/licenses/by-nc-nd/3.0/ 